\title{
THE COMPARISON OF NUCLEAR UBIQUITOUS CASEIN AND CYCLIN-DEPENDENT KINASES SUBSTRATE (NUCKS) WITH Ki67 PROLIFERATION MARKER EXPRESSION IN COMMON SKIN TUMORS
}

\author{
Krzysztof Zduniak, Siddarth Agrawal, Krzysztof Symonowicz, Kamil Jurczyszyn, \\ Piotr ZióŁKowsKi
}

Department of Pathology, Wroclaw Medical University, Wrocław, Poland

\begin{abstract}
Nuclear ubiquitous casein and cyclin-dependent kinases substrate (NUCKS) is a chromosomal protein of unknown function. Its amino acid composition and structure of its DNA binding domain resemble those of high mobility group A (HMGA) proteins which are associated with various malignancies. Since changes in expression of HMGA are considered as a marker of tumor progression, it is possible that similar changes in expression of NUCKS could be a useful tool in diagnosis of malignant skin tumors. To investigate this assumption we used specific antibodies against NUCKS for immunohistochemistry of squamous (SCC) and basal cell carcinoma (BCC) as well as keratoacanthoma (KA). We found high expression of NUCKS in nuclei of SCC and BCC cells which exceeded expression of the well-known proliferation marker Ki67. Expression of NUCKS in benign KA was much below that of malignant tumors. With the present study and based on our previous experience we would like to suggest the NUCKS protein as a novel proliferation marker for immunohistochemical evaluation of formalin-fixed and paraffin-embedded skin tumor specimens. We would like to emphasize that NUCKS abundance in malignant skin tumors is higher than that of the well-known proliferation marker Ki67, thus allowing more precise assessment of tumor proliferation potential.
\end{abstract}

Key words: squamous cell carcinoma, basal cell carcinoma, keratoacanthoma, Ki67, NUCKS.

\section{Introduction}

Nuclear ubiquitous casein and cyclin-dependent kinases substrate (NUCKS) is a nuclear DNA binding protein occurring in almost all types of human cells $[1,2]$. Its biological functions are not well understood, but its structural similarity to the high mobility group A (HMGA) proteins suggests that it may play a role in regulation of chromatin structure and its activity [3-5].

The abundance of NUCKS in rapidly growing cells as well as the overexpression of nucks mRNA in ovarian cancer [6] suggests that this protein may be involved in facilitating and maintaining activity of transcription of some genes during rapid proliferation and in cancer.
Until now NUCKS has been studied in detail using a variety of biochemical and cell biological methods $[1$, $2,7-11]$. Some of these analyses related the occurrence of the protein to histological grade or to a particular tumor type and also to benign epithelial proliferations. Several studies performed during the last four years by independent research groups have revealed similar results in relation to significance of NUCKS expression in a number of breast lesions [7, 11]. Using patient-matched samples of normal breast tissue and breast cancer by mass spectrometry we revealed elevated levels of NUCKS in protein extracts from ductal breast cancers [11]. Immunohistochemistry using polyclonal antibodies raised against a NUCKS-relat- 
ed peptide revealed elevated expression of NUCKS in the cells of invasive ductal carcinoma. Statistically significant differences between groups of $\mathrm{G} 1$ and G2, and also between $\mathrm{G} 1$ and $\mathrm{G} 3$ cancers were confirmed, whereas no significant difference between groups G2 and G3 was found [11]. The elevated expression of the NUCKS gene was also found to be associated with high-invasive phenotype in mouse lung adenocarcinoma cell strains and the NUCKS protein was increased in the majority of the cell strains [12]. Additionally Kikuchi et al. revealed a significant correlation between NUCKS expression level and tumor stage, CEA level and other features in patients with colorectal carcinoma $[13]$.

The expression of $\mathrm{Ki} 67$ protein is closely associated with cell proliferation and this has been examined in the past in various malignant tumors including breast cancer [14]. The antigen can be exclusively detected within the nucleus during the interphase, whereas in mitosis it is relocated to the surface of the chromosomes. The fact that Ki67 protein is present during all active phases of the cell cycle and mitosis, but is absent in resting cells, makes it an excellent marker for determining the growth fraction of a given cell population [15]. Immunohistochemical analysis of antigens present within the targeted cancer tissue remains the main diagnostic procedure worldwide. It is obvious because the routine immunohistochemistry is a simple, not expensive and a relatively fast method of diagnosis. SCC is recognized as a malignant, metastasizing tumor, often with poor prognosis, whereas $\mathrm{BCC}$ is a locally malignant and non-metastasizing tumor with good prognosis. BCC is reported as the most frequent malignant tumor in human patients. An interesting review about basal cell carcinoma (BCC) has recently been published by Mackiewicz-Wysocka et al. [16]. Therefore squamous cell carcinoma (SCC) and BCC might be considered convenient and easily available models for basic studies in oncology. Both are routinely and usually successfully treated by surgical excision and in SCCs also by radiotherapy (stage I or II), supported by chemo-radiation in stage III and IV [17]. Progress has been made in the development of new treatment methods, especially in treatment of SCCs, in the last decades. Although these methods resulted in general improvement of therapy outcomes, the 5-year survival rates were not significantly improved [18]. Therefore, the early diagnosis of primary malignant tumors and the search for new markers of cell proliferation remain challenging tasks.

In this work we analyzed the occurrence of NUCKS in cells of the two most common skin tumors, squamous and basal cell carcinoma (SCC and BCC), and in benign keratoacanthoma (KA) in relation to expression of a well-known proliferation marker, Ki67. To our knowledge, this is the first study ana- lyzing occurrence of NUCKS in SCC, BCC and KA cells in relation to Ki67.

\section{Material and methods}

\section{Antibodies}

Antibodies against NUCKS were prepared accordingly to the procedure described previously [11]. Polyclonal anti-Ki67 was purchased from Novocastra, UK (NCL-Ki67p) and the staining was performed in the same way as in the case of NUCKS.

\section{Histopathology}

Seventy-eight cases of clinically suspected solitary skin tumors were included in the present study. They were obtained between January and December 2012, collected at the Department of Pathology, Wroclaw Medical University, from surgical units in Lower Silesia, Poland. Tumors were excised using a standard surgical protocol with wide margins of macroscopically unaltered skin. All other cases with incomplete excision or greatly disintegrated were excluded from the study. Local Ethics Committee guidelines were followed.

The samples from 78 tumors were fixed in $4 \%$ formalin and embedded in paraffin. After that, the paraffin blocks were cut on a microtome to obtain $4 \mu \mathrm{m}$ thick slices which were mounted on glass slides and stained with hematoxylin-eosin (HE) or with antibodies. Evaluation of HE-stained samples was performed by use of a light microscope (OLYMPUS BX50) at magnification 100 or 200 times. This microscopic evaluation confirmed basal cell carcinoma in 30 and squamous cell carcinoma in 27 cases, and benign keratoacanthoma in 21 cases. We chose the above 3 tumors because they represent three different ways of tumor growth: keratoacanthoma is benign; basal cell carcinoma is locally malignant and non-metastasizing; while squamous cell carcinoma is malignant and metastasizing.

Table I shows the general characteristics of BCC and SCC, and KA cases examined in this study.

\section{Immunohistochemical staining}

Following deparaffinization the antigen determinant was retrieved using normal pressure cooking in $0.01 \mathrm{M}$ sodium citrate for 9 minutes in a $350 \mathrm{~W} \mathrm{mi-}$ crowave oven at $\mathrm{pH} 6$. The slides were blocked for endogenous peroxidase (Peroxidase Blocking Reagent, DAKO Cytomation) and incubated overnight with anti-NUCKS antibodies at a concentration of $15 \mu \mathrm{g} /$ $\mathrm{ml}$ in PBS containing 125-fold diluted swine serum at $4^{\circ} \mathrm{C}$. The binding of the primary antibodies was visualized using the $A B C$ method (kit LSAB, DAKO Cytomation) and stained with DAB (DAKO). The PBS was used at each step. Finally, the slides were 
Table I. General characteristics of basal cell carcinoma (BCC) and squamous cell carcinoma (SCC) and keratoacanthoma in relation to localization

\begin{tabular}{lccccccc}
\hline & Nose & Eyelid & Forehead & Cheek & Ear & Other & Total \\
\hline Nodular BCC & 7 & 1 & 4 & 3 & 3 & 0 & 18 \\
\hline Multifocal superficial BCC & 2 & 1 & 3 & 4 & 2 & 0 & 12 \\
\hline Keratinizing SCC & 5 & 0 & 1 & 3 & 4 & 8 & 21 \\
\hline Non-keratinizing SCC & 2 & 0 & 1 & 1 & 0 & 2 & 6 \\
\hline Keratoacanthoma & 4 & 0 & 1 & 2 & 2 & 12 & 21 \\
\hline
\end{tabular}

counterstained with hematoxylin and rinsed in tap water.

The percentage of positively stained tumor cells was evaluated on 5 fields from the center of the tumor: the number of positively stained cells (nuclei) was divided by the total number of cancer cells seen on that field at magnification of 400 times. A mean value from 5 such fields is shown in the "results" section. The selection criterion for the 5 fields measured was the center of each sample and this was assessed by two independent pathologists. The intensity of staining was evaluated as: none $(\mathrm{N})$, low $(\mathrm{L})$, low-to-intermediate (LI), intermediate (IN), intermediate-to-high (IH) or high $(\mathrm{H})$.

The positive control for NUCKS and Ki67 antibodies was obtained by using samples of invasive ductal carcinoma (IDC) where both were found to result in strong positive reactions. The negative control was obtained by omitting the primary antibody. We also checked whether inflammatory cells, such as lymphocytes or plasma cells, expressed NUCKS.

The microphotographs were taken using a light microscope (OLYMPUS BX40) at magnification 200 times and a digital camera (OLYMPUS DP10). Statistical analysis of the correlation between number of positively stained cells and histological type of examined tumors was made. One-way analysis of variance (ANOVA) by ranks was used for statistical analysis.

\section{Results}

Immunohistochemical analysis of NUCKS in BCC and SCC, and in KA.

All samples were first evaluated with regard to their histological type (nodular or multifocal, superficial BCC, SCC or KA) and then the samples from each tumor were studied using an immunohistochemical method. The occurrence of NUCKS was observed in $87.8 \%$ of nuclei of tumor cells in BCCs (mean value from all examined cases) (Fig. 1A and Table II), and to lesser extent, and in some cases only, in endothelia, lymphocytes and in plasma cells. The intensity of staining of BCC cells was evaluated as intermediate-to-high. The cells of BCC revealed high proliferation activity and the staining for $\mathrm{Ki} 67$ was found to be positive in almost $47 \%$ of cells from all examined cases (Fig. 1B and Table II). The intensity of staining was evaluated as IH.

The staining for NUCKS in cells of SCC turned out to be comparable to that of BCC. The staining revealed an $\mathrm{IH}$ or high intensity and the occurrence of NUCKS was observed in nuclei of $83.85 \%$ of all examined cancer cells. Figure $1 \mathrm{C}$ shows the positive staining for NUCKS in the cells of SCC. In that tumor the occurrence of NUCKS also exceeded $80 \%$ (in particular $-82 \%$ ) (see also Table II). Positive staining was also observed in a small number of other cells such as lymphocytes or endothelial cells. Comparison of NUCKS expression between the BCC, SCC and $\mathrm{KA}$ is shown in Fig. 2A. Percentage of the NUCKS expression is different in each group $(\mathrm{p}<0.05)$.

The percentage of $\mathrm{Ki} 67$ expression in BCC, SCC and $\mathrm{KA}$ group is shown in Fig. 2B; statistical analysis revealed differences between each group $(p<0.05)$. The positive staining for Ki67 in the cells of SCC was found in on average $72.3 \%$ of cells from all cases (see Table II). The intensity of positive staining was assessed as IH (Fig. 1D). In general, the cells of SCC were found to express positive reaction for $\mathrm{Ki} 67$ at higher frequency than the cells of BCC. Staining for NUCKS in the cells from the benign tumor (KA) was clearly less frequent than that of SCC or of BCC. Figure $1 \mathrm{E}$ shows the result of immunostaining against NUCKS in one case of KA, where it was found in more than $50 \%$ of nuclei of tumor cells. The average of the positive cellular staining for the whole group of KA was $53.5 \%$ and the intensity as LI. Similarly to above two cancers, a small number of lymphocytes, plasma cells and endothelial cells expressed NUCKS.

In contrast to SCC or to BCC, the staining for $\mathrm{Ki} 67$ in the cells of KA turned out to be very weak: only basal cells from the epithelium showed positive reaction. The intensity was evaluated as $\mathrm{IH}$ and the frequency did not exceed $20 \%$ (mean: $17.8 \%$ for all examined KAs) (Fig. 1F, Table II).

\section{Discussion}

The BCC, SCC and KA belong to the most frequent tumors in humans. The incidence of $\mathrm{BCC}$ in the skin was proved to be the highest among all malignant skin tumors. Although the BCC was classi- 

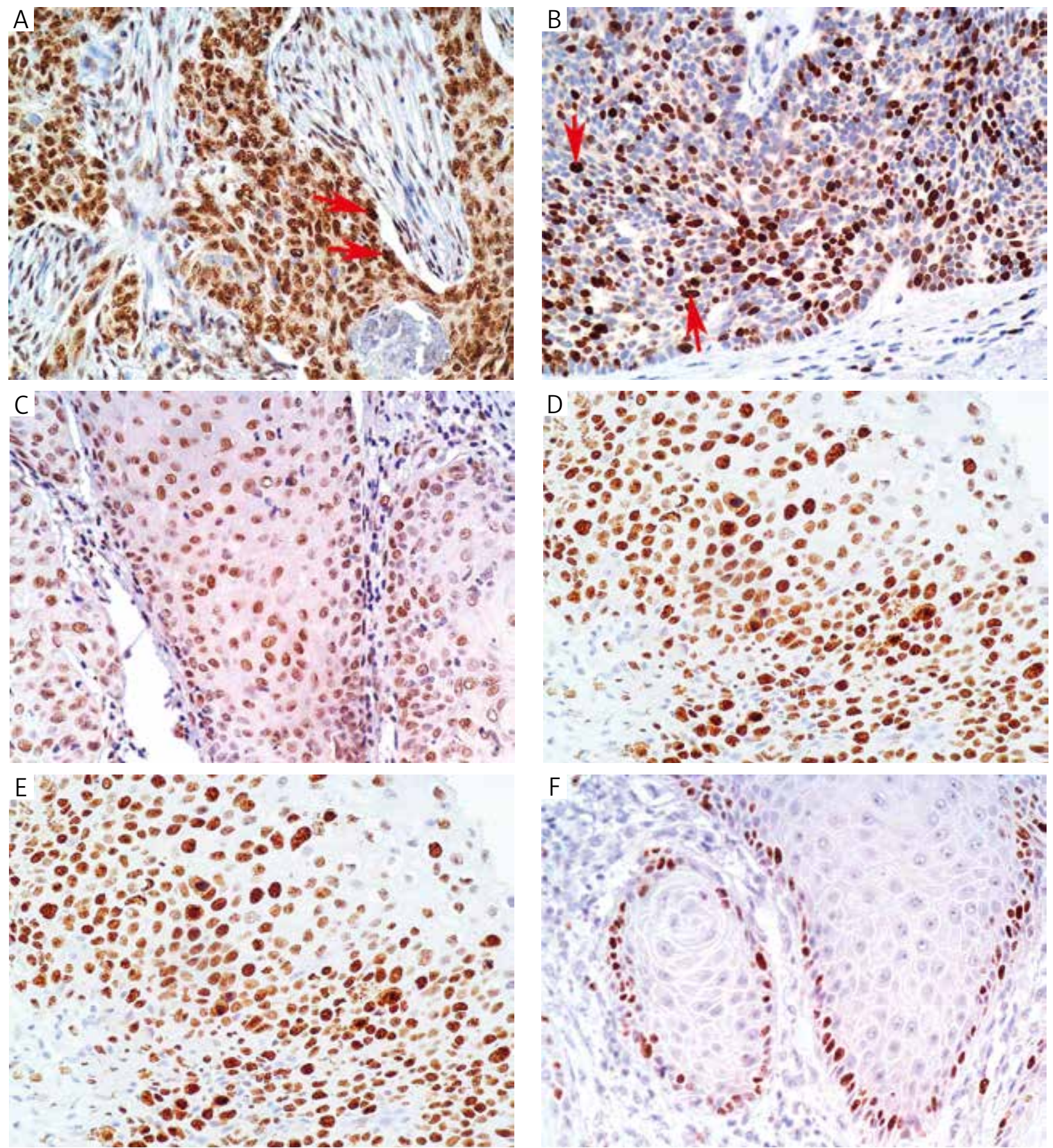

Fig. 1. A) Distinct nuclear expression of NUCKS in the cells of basal cell carcinoma of the skin (multifocal, superficial type of nasal skin). Positive staining was observed in over $90 \%$ of cancer cells in this case; intensity - IH; arrows show NUCKS in nuclei of BCC cells. B) Positive staining for Ki67 within cells of basal cell carcinoma of the skin, multifocal, superficial type of nasal skin. Positive staining was found here in almost $48 \%$ of cancer cells (arrows); the intensity of staining - IH. The same case as in Fig. 1A. C) Distinct nuclear expression of NUCKS in the cells of squamous cell carcinoma of the skin. Positive staining was observed in $82 \%$ of cancer cells in that case; the intensity was evaluated as intermediate-to-high (IH). D) Distinct nuclear expression of Ki67 in the cells of squamous cell carcinoma of the skin. Positive staining was observed in $74 \%$ of cancer cells in that case; the intensity was evaluated as intermediate-to-high (IH). E) The staining for NUCKS in the cells of keratoacanthoma. Positive staining was observed in $55 \%$ of tumor cells in that case; the intensity was evaluated as low-to-intermediate (LI). F) Distinct nuclear expression of Ki67 in the cells of keratoacanthoma seen in the basal layer. Positive staining was observed in $18 \%$ of tumor cells in that case; the intensity was evaluated as intermediate-to high $(\mathrm{IH}) ; \mathrm{ABC}$ method was used in all above cases, magnification $200 \times$; hematoxylin counterstaining 
Table II. Immunohistochemical expression of NUCKS (columns 3 and 4) and of Ki67 (columns 5 and 6) in the cells of basal cell carcinoma, squamous cell carcinoma and keratoacanthoma in $\%$

\begin{tabular}{lccccc}
\hline & \multicolumn{2}{c}{ NUCKS } & \multicolumn{2}{c}{ KI67 } \\
\cline { 2 - 7 } & TotAL & MeAN $(\%)$ & SD & MeAn (\%) & SD \\
\hline Keratoacanthoma & 21 & 53.5 & 6.1 & 17.8 & 5.2 \\
\hline Basal cell carcinoma & 30 & 87.8 & 5.6 & 46.7 & 7.1 \\
\hline Squamous cell carcinoma & 27 & 83.8 & 6.3 & 72.3 & 7.5 \\
\hline NUCKS - nuclear ubiquitous casein and cyclin-dependent kinases substrate, SD - standard deviation & & &
\end{tabular}

A

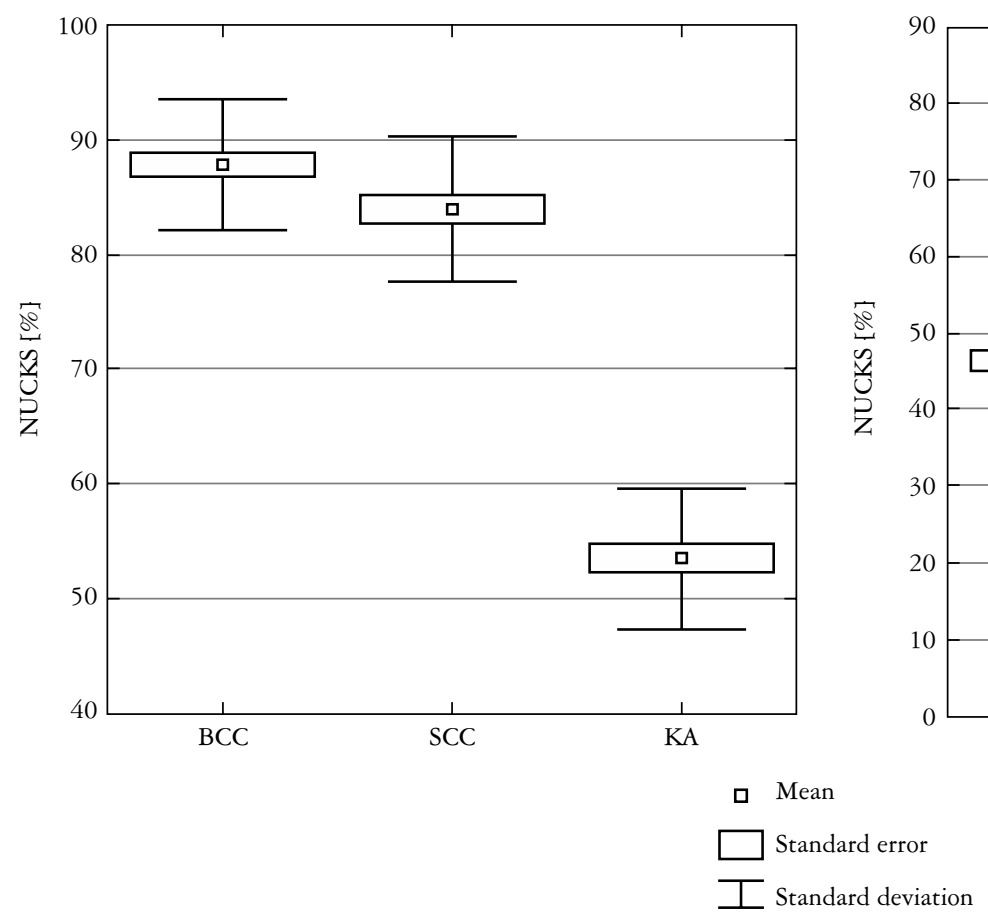

B

Fig. 2. A) Comparison of NUCKS expression between basal cell carcinoma (BCC), squamous cell carcinoma (SCC) and keratoacanthoma (KA). Y-axis percentage of positive immunohistochemical staining, X-axis compared groups. B) Comparison of Ki67 expression between basal cell carcinoma (BCC), squamous cell carcinoma (SCC) and keratoacanthoma (KA). Y-axis percentage of positive immunohistochemical staining, $\mathrm{X}$-axis compared groups

fied as locally malignant carcinoma, without metastatic spread, the tumor can relapse after incomplete surgical excision. Thus, the cases of recurrent BCC are very frequently observed. Squamous cell carcinoma in turn, belongs to the malignant tumors with the largest dissemination within the human body. Squamous cell carcinoma occurs in every localization where the squamous epithelium is present, e.g. in larynx, esophagus, uterine cervix, oral mucosa and in the skin. The $\mathrm{KA}$ is not very common lesion and it is often misdiagnosed as a malignant condition. It is even claimed that KA may lead to SCC [19]. We chose these three tumors as model tumors for our studies to investigate a correlation of occurrence between NUCKS and the well known proliferation marker, Ki67, because they represent three differ- ent ways of tumor growth. Namely, KA is a benign, $\mathrm{BCC}$ - locally malignant, non-metastasizing and the SCC - malignant and metastasizing tumor. In order to differentiate SCC from KA a panel of antibodies including p53, Ki67 and bcl-2 was already applied in previous studies [20]. The immunohistochemical staining revealed that the presence of p53, Ki67 and $\alpha$-SMA was associated with more aggressive behavior in large BCC [21]. The expression of p63 in skin tumors, i.e. in BCC, SCC and in KA to clarify its role in the development and differential diagnosis of these lesions, was also investigated [22]. p63 immunostaining was also used in distinguishing between other malignant and benign skin tumors [23]. The other search for markers to be applied in SCC diagnosis revealed that there is a panel of markers that might be 
used in practice. Strong cytoplasmic staining of receptor activator of NF- $\mathrm{KB}$ ligand (RANKL) proteins was detected in cells of both buccal and gingival SCC. A similar staining pattern was noted for RANK protein in these localizations while an absence of staining of RANK protein was observed in all healthy tissues [24]. The role of EGFR [25] or miR-205 and miR-21 in SCC diagnosis was raised in another study [26]. In other study, Bcl-x immunoreactivity was found in $88 \%$ of SCC, whereas in KA it was typically confined to the mid-to-upper spinous keratinocytes and generally found in $59 \%$ suggesting that the protein contributes to the survival advantage and aggressiveness of the SCC [27]. Class III $\beta$-tubulin expression was demonstrated in $50 \%$ of BCCs and it was suggested as a useful marker for distinguishing BCC from non-neoplastic hair buds [28], while in our study the expression of NUCKS in BCCs was much higher than that of III $\beta$. CD10 is considered to be useful for distinguishing BCC (especially superficial BCC) and SCC in difficult cases [29, 30], also it might be helpful for differentiating between BCC and some benign skin tumors [29]. In our study the expression of above mentioned $\mathrm{Ki} 67$, which reflects the malignancy potentials, was strongest in SCC $(72.3 \%)$, lower in BCC $(47 \%)$, while in KA it did not exceed $18 \%$. Within the present study we also carried out parallel immunohistochemical observations which proved that NUCKS is overexpressed in cells of BCC and in SCC in similar proportions as this was found in IDC in our previous study, i.e. $87.8 \%$ vs. $83.85 \%$ in BCC and SCC, respectively. The expression of NUCKS in the cells of KA was significantly much below than that in two above cancers, i.e. $53.5 \%$. The NUCKS expression was found in nuclei of tumor cells, similarly to the staining pattern for NUCKS in IDC. The staining pattern for NUCKS in both skin cancers was very similar, i.e. it was evenly disseminated throughout the whole tumor and the intensity of staining was intermediate-to-high or intermediate, whereas in KAs with disseminated staining pattern the intensity was low-to-intermediate or low. This supports the assumption that stronger NUCKS expression may reflect higher malignancy potential of examined tumor. Drosos et al. [7] found that the expression of NUCKS did not show correlation with Ki67 expression, neither in different breast lesions, including malignant tumors, nor in cell cultures. In our present study we found that the strong expression of NUCKS was observed in larger amount of cells than the expression of Ki67 and this referred to all examined skin tumors. The overexpression of NUCKS in the basal cell and SCC cells could then better point towards its relation with malignant potentials of the tumor, i.e. potentials for invasion and metastasis than the expression of Ki67. This finding could be supported by the results showing that elevated expression of
NUCKS gene is associated with high-invasive phenotype in a mouse lung adenocarcinoma cell strains and the NUCKS protein is increased in majority of the cell strains [12]. Accordingly to Mantovani [31] cancer can be characterized by six hallmarks including the ability to invade other tissues and inflammation. Chechlinska et al. [32] emphasized that cancer and inflammation are inextricably linked and that the cancer patients have local and systemic changes in inflammatory parameters. They suggested that every new biomarker for cancer studies should be then evaluated with respect to inflammation in order to exclude inflammatory reaction in cancer patient, probably because activated lymphocytes express numerous genes regarded as tumor-specific markers. In our present study we observed a very weak NUCKS expression in single lymphocytes, endothelial cells and neither in macrophages nor in plasma cells. In all examined cases of BCC, SCC and KA the expression of NUCKS was found entirely in single inflammatory cells. Thus, we believe that the expression of NUCKS in different types of skin tumors should be considered as biomarker for tumor malignancy and not for inflammation. This remains in agreement with general findings of the other authors [7] where the most intense NUCKS staining was found in invasive ductal carcinoma compared to normal tissues.

In summary, we would like to emphasize that NUCKS is overexpressed in BCC, SCC cells and it is also expressed in KA cells showing much higher expression than that of Ki67. Based on the present and previous studies we would like to suggest the NUCKS as novel tumor marker in immunohistochemical evaluation of formalin-fixed and paraffin-embedded BCC and SCC specimen although further investigations are required to evaluate its role in histological diagnosis.

\section{Acknowledgements}

We would like to thank Prof. Jacek Wisniewski for supplying us with antibody against NUCKS.

Authors declare no conflict of interest.

\section{References}

1. Grundt K, Haga IV, Aleporou-Marinou V, et al. Characterisation of the NUCKS gene on human chromosome 1q32.1 and the presence of a homologous gene in different species. Biochem Biophys Res Commun 2004; 323: 796-801.

2. Ostvold AC, Norum JH, Mathiesen S, et al. Molecular cloning of a mammalian nuclear phosphoprotein NUCKS, which serves as a substrate for Cdk1 in vivo. Eur J Biochem 2001; 268: 2430-2440.

3. Wisniewski JR, Schwanbeck R. High mobility group I/Y: multifunctional chromosomal proteins causally involved in tumor 
progression and malignant transformation (review). Int J Mol Med 2000; 6: 409-419.

4. Sgarra R, Zammitti S, Lo Sardo A, et al. HMGA molecular network: from transcriptional regulation to chromatin remodeling. Biochim Biophys Acta 2010; 1799: 37-47.

5. Sgarra R, Rustighi A, Tessari MA, et al. Nuclear phosphoproteins HMGA and their relationship with chromatin structure and cancer. FEBS Lett 2004; 574: 1-8.

6. Schaner ME, Ross DT, Ciaravino G, et al. Gene expression patterns in ovarian carcinomas. Mol Biol Cell 2003; 14: 43764386.

7. Drosos Y, Kouloukoussa M, Østvold AC, et al. NUCKS overexpression in breast cancer. Cancer Cell Int 2009; 10: 19.

8. Grundt K, Skjeldal L, Anthonsen HW, et al. A putative DNA-binding domain in the NUCKS protein. Arch Biochem Biophys 2002; 407: 168-175.

9. Grundt K, Haga IV, Huitfeldt HS, Ostvold AC. Identification and characterization of two putative nuclear localization signals (NLS) in the DNA-binding protein NUCKS. Biochim Biophys Acta 2007; 1773: 1398-1406.

10. Wiśniewski JR, Zougman A, Krüger S, et al. Constitutive and dynamic phosphorylation and acetylation sites on NUCKS a hypermodified nuclear protein, studied by quantitative proteomics. Proteins 2008; 73: 710-718.

11. Ziółkowski P, Gamian E, Osiecka B, et al. Immunohistochemical and proteomic evaluation of nuclear ubiquitous casein and cyclin-dependent kinases substrate in invasive ductal carcinoma of the breast. J Biomed Biotechnol 2009; 2009: 919645.

12. Sargent LM, Ensell MX, Ostvold AC, et al. Chromosomal changes in high- and low-invasive mouse lung adenocarcinoma cell strains derived from early passage mouse lung adenocarcinoma cell strains. Toxicol Appl Pharmacol 2008; 233: 81-91

13. Kikuchi A, Ishikawa T, Mogushi K, et al. Identification of NUCKS1 as a colorectal cancer prognostic marker through integrated expression and copy number analysis. Int J Cancer 2013; 132: 2295-2302.

14. Kuenen-Boumeester V, Van Der Kwast TH, Van Laarhoven HA, Henzen-Logmans SC. Ki-67 staining in histological subtypes of breast carcinoma and fine needle aspiration smears. J Clin Pathol 1991; 44: 208-210.

15. Scholzen T, Gerdes J. The Ki-67 protein: from the known and the unknown. J Cell Physiol 2000; 182: 311-322.

16. Mackiewicz-Wysocka M, Bowszyc-Dmochowska M, Strzelecka-Węklar D, et al. Basal cell carcinoma - diagnosis. Contemp Oncol 2013; 17: 337-342.

17. Deo SV, Hazarika S, Shukla NK, et al. Surgical management of skin cancers: experience from a regional cancer centre in North India. Indian J Cancer 2005; 42: 145-150.

18. Lam L, Logan RM, Luke C, Rees GL. Retrospective study of survival and treatment pattern in a cohort of patients with oral and oropharyngeal tongue cancers from 1987 to 2004. Oral Oncol 2007; 43: 150-158.

19. Sánchez Yus E, Simón P, Requena L, et al. Solitary keratoacanthoma: a self-healing proliferation that frequently becomes malignant. Am J Dermatopathol 2000; 22: 305-310.

20. Connolly M, Narayan S, Oxley J, de Berker DA. Immunohistochemical staining for the differentiation of subungual keratoacanthoma from subungual squamous cell carcinoma. Clin Exp Dermatol 2008; 33: 625-628.

21. Pilloni L, Bianco P, Manieli C, et al. Immunoreactivity for alpha-smooth muscle actin characterizes a potentially aggressive subgroup of little basal cell carcinomas. Eur J Histochem 2009; 53: 113-116.

22. Sakiz D, Turkmenoglu TT, Kabukcuoglu F. The expression of p63 and p53 in keratoacanthoma and intraepidermal and invasive neoplasms of the skin. Pathol Res Pract 2009; 205: 589-594.
23. Vidal CI, Goldberg M, Burstein DE, et al. p63 Immunohistochemistry is a useful adjunct in distinguishing sclerosing cutaneous tumors. Am J Dermatopathol 2010; 32: 257-261.

24. Chuang FH, Hsue SS, Wu CW, Chen YK. Immunohistochemical expression of RANKL, RANK, and OPG in human oral squamous cell carcinoma. J Oral Pathol Med 2009; 38: 753-758.

25. Bernardes VF, Gleber-Netto FO, Sousa SF, et al. Clinical significance of EGFR, Her-2 and EGF in oral squamous cell carcinoma: a case control study. J Exp Clin Cancer Res 2010; 29: 40.

26. Kimura S, Naganuma S, Susuki D, et al. Expression of microRNAs in squamous cell carcinoma of human head and neck and the esophagus: miR-205 and miR-21 are specific markers for HNSCC and ESCC. Oncol Rep 2010; 23: 1625-1633.

27. Tan KB, Lee YS. Immunoexpression of $\mathrm{Bcl}-\mathrm{x}$ in squamous cell carcinoma and keratoacanthoma: differences in pattern and correlation with pathobiology. Histopathology 2009; 55 : 338-345.

28. Ishida M, Kushima R, Okabe H. Aberrant expression of class III $\beta$-tubulin in basal cell carcinoma of the skin. Oncol Rep 2009; 22: 733-737.

29. Sari Aslani F, Akbarzadeh-Jahromi M, Jowkar F. Value of CD10 expression in differentiating cutaneous basal from squamous cell carcinomas and basal cell carcinoma from trichoepithelioma. Iran J Med Sci 2013; 38: 100-106.

30. Wagoner J, Keehn C, Morgan MB. CD-10 immunostaining differentiates superficial basal cell carcinoma from cutaneous squamous cell carcinoma. Am J Dermatopathol 2007; 29: 555-558.

31. Mantovani A. Cancer: inflaming metastasis. Nature 2009; 457: 36-37.

32. Chechlinska M, Kowalewska M, Nowak R. Systemic inflammation as a confounding factor in cancer biomarker discovery and validation. Nat Rev Cancer 2010; 10: 2-3.

\section{Address for correspondence}

Prof. dr Piotr Ziółkowski

Department of Pathology

Wroclaw Medical University

1 Marcinkowskiego

50-368 Wrocław, Poland

tel. +48717841212

fax +48717840057

e-mail: ziolkows@interia.pl 